\title{
MACHINE VISION BASED COTTON RECOGNITION FOR COTTON HARVESTING ROBOT
}

\author{
Yong Wang ${ }^{1,2}$, Xiaorong $\mathrm{Zhu}^{3}$, Changying $\mathrm{Ji}^{1{ }^{1, *}}$ \\ ${ }^{1}$ College of Engineering, Nanjing Agricultural University, Nanjing, China, 210031 \\ ${ }^{2}$ College of Sciences, PLA University of Science and Technology, Nanjing, China, 210007 \\ ${ }^{3}$ Department of Radio Engineering of South East University, Nanjing, China, 210049 \\ *Corresponding author, Address: College of Engineering, Nanjing Agricultural University, \\ Nanjing, 210031, P. R. China, Tel: +86-10-58766570,Email: chyji@njau.edu.cn
}

\begin{abstract}
A new cotton recognition method is proposed in this paper. It provides parameters for motion of the manipulator so that it can acquire precise location information of cotton, identify cotton from surroundings correctly, and accordingly pick up them automatically. This method is based on color subtraction information of different parts of cotton. Furthermore, in order to increase accuracy rate of cotton recognition, dynamic Freeman chain coding is used to remove noise. Experimental results show that the proposed method has good performance for cotton identification.
\end{abstract}

Keywords: cotton recognition, image processing, Freeman chain coding

\section{INTRODUCTION}

Agricultural automation and intelligence have become a major issue in recent years (Shiqchil et al., 2001; Alessio et al., 2001; Slaughter et al., 1999). However, autonomous harvesting robots have not yet been commercially applied in horticulture practices. Obviously, the price and performance of harvesting robots have not yet met the requirements for their successful introduction in harvesting practices (Yud-Ren et al., 2002; Sander, 2005; VanHenten et al., 2003; Elias et al., 2003; Tadhg et al., 2004). In this 
paper, a new cotton recognition method is introduced, which is based on color subtraction information. Besides, the modified dynamic Freeman chain coding is used to further increase recognition accuracy.

\section{COLOR ANALYSIS FOR COTTON}

In image processing, there are six color spaces widely used, namely, RGB, normalized rgb, HIS, YCrCb, $\mathrm{L}^{*} \mathrm{a}^{*} \mathrm{~b}^{*}$ and $\mathrm{I}_{1} \mathrm{I}_{2} \mathrm{I}_{3}$. The objects investigated are four hundred images of cotton taken in Jiangpu Farm of Nanjing Agricultural University from ten to twelve in the morning and from two to four in the afternoon from October to November in sunshiny weather. Each image is 24-bit true-color. Color data of cotton fruits, leaves and stems are extracted from these images. According to transition formulas of different color space (Stephen et al., 2003), color mean distributions of them in six color space models are respectively drawn as shown in Figure 1.

Although $\mathrm{I}_{1} \mathrm{I}_{2} \mathrm{I}_{3}$ and $\mathrm{L}^{*} \mathrm{a}$ " $\mathrm{b}$ " color space can be used to identify cotton fruits from background, they need much time to convert from RGB module to other spaces. In general, none of the six color space can precisely identify cotton fruit from the background in real-time.
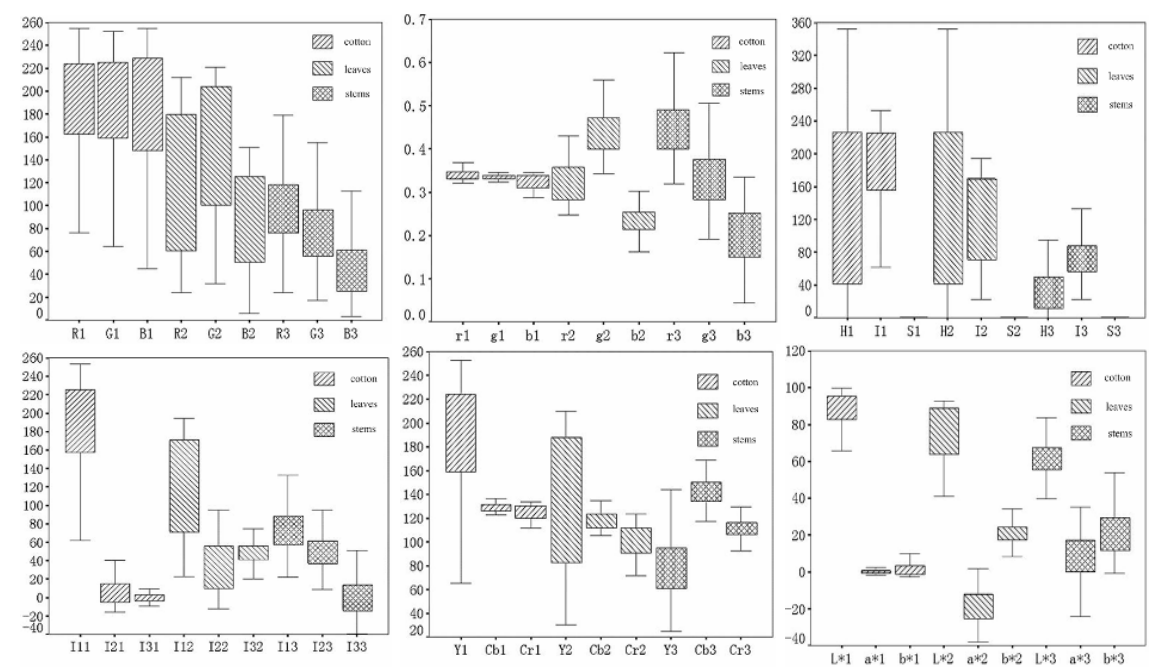

Fig. 1. Mean distribution of color of cottons, leaves and stems in six color models 


\section{IMAGE PROCESSING ALGORITHM FOR COTTON RECOGNITION}

\subsection{Color Subtraction Module}

In order to correctly identify cotton fruits from the background in realtime, a new color module was developed. According to above analysis, it can be seen that the color values of red, green and blue of cotton fruits are quite the same, whereas other two are quite different. Hence, this character can be used to differentiate cotton fruits from the other two. The distribution of redblue $(R-B)$, red-green $(R-G)$ and green-blue $(G-B)$ of cotton fruits, leaves and stems are shown in Figure 2.

In this paper the subtraction value of red and blue is selected. And the algorithm is as follows:

$$
g(x, y)= \begin{cases}0, & \text { if } f(x, y)<T \\ 255, & \text { otherwise }\end{cases}
$$

where $f(x, y)$ is the gray value of pixel $(x, y)$, and $T$ is the threshold. As example, Figure 3(b) is recognition result by color subtraction module.
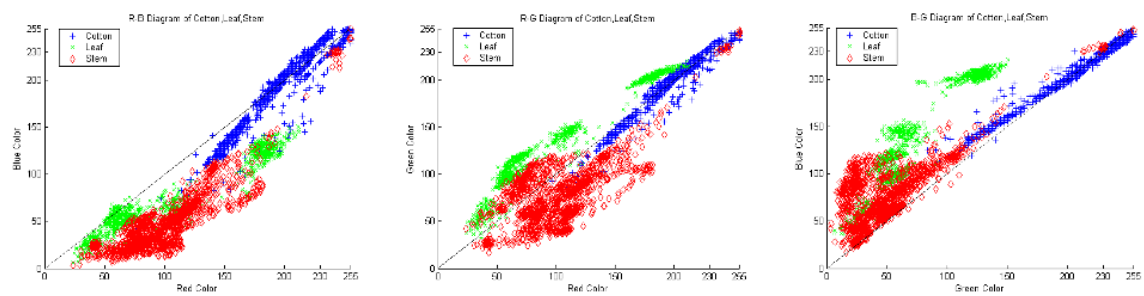

Fig. 2. Distribution of R-B, R-G and G-B

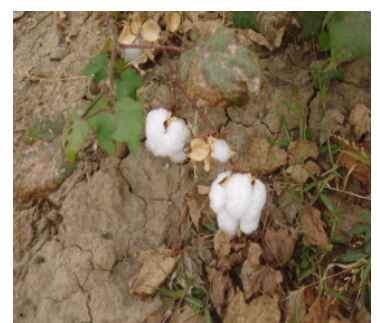

(a)

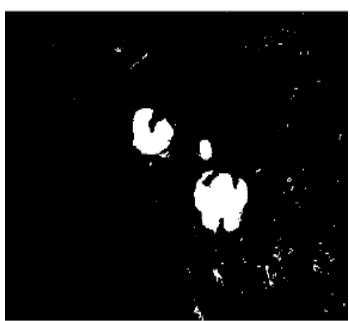

(b)

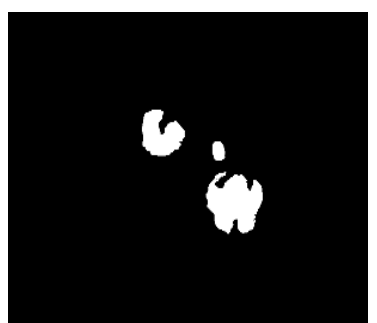

(c)

Fig. 3. Original image and recognition results

\subsection{Dynamic Freeman Chain Coding}

In order to solve the white points in Figure 3(b), a new method of dynamic Freeman chain coding is proposed. 
The algorithm of dynamic Freeman chain coding is as following:

(1) Scan the binary image, if the pixel value is 255 , then labeled, and use the rule of Freeman to trace anticlockwise.

(2) Compute the perimeters of different regions.

(3) Remove the region which perimeter is less than $\mathrm{T}$ which is calculated by the formula:

$$
\begin{aligned}
& T=\mu_{1} P_{1}+\mu_{2} P_{2} \\
& \mu_{1}=\frac{M 1}{\sum_{i=1}^{n} \text { perimeter }}, \mu_{2}=1-\mu_{1}
\end{aligned}
$$

where $\mathrm{P}_{1}$ is the median value of perimeter, $\mathrm{P}_{2}$ is the maximum value of perimeter, $\mu_{1}$ and $\mu_{2}$ are weight indexes, $M_{1}$ is the number which perimeter is less than the median value. If perimeter is less than $\mathrm{T}$, the value of the pixel in the region is 0 , otherwise 255 .

Figure 3(c) shows the recognition result by dynamic Freeman coding.

\section{PERFORMANCE EVALUATION}

In this section, we evaluate the image processing algorithm for cotton recognition by experiments. Table 1 shows the experimental results. It shows that accuracy of recognition reaches above $85 \%$ in the four experiments.

Table 1. Recognition results for cotton fruits

\begin{tabular}{lccc}
\hline $\begin{array}{l}\text { Number of } \\
\text { images }\end{array}$ & $\begin{array}{l}\text { Number of cotton } \\
\text { fruits }\end{array}$ & $\begin{array}{l}\text { Recognition results by } \\
\text { Freeman coding }\end{array}$ & Accuracy (\%) \\
\hline 20 & 42 & 37 & 88.09 \\
50 & 101 & 87 & 86.14 \\
100 & 182 & 158 & 86.81 \\
150 & 273 & 237 & 86.81 \\
\hline
\end{tabular}

\section{CONCLUSION}

A new cotton recognition method is proposed, which is based on color subtraction of different parts of cotton. Besides, dynamic Freeman chain coding is used to increase recognition accuracy. Experimental results show that the method has high performance for no-overlapping individual cotton recognition. 


\section{REFERENCES}

Alessio Plebe, Giorgio Grasso. Localization of spherical fruits for robotic harvesting. Machine Vision and Application, (2001) 13:70-79

E.J. Van Henten, B.A.J. Van Tuijl, J. Hemming, et al. Field Test of an Autonomous cucumber picking robot. Biosystem Engineering (2003) 86(3):305-313

Elias N. Malamas, Euripides G.M. Petrakis, Michalis Zervakis, et al. A survy on industrial vision systems, applications and tools. Image and Vision Computing 21 (2003): 171-188

K.F. Sanders. Orange Harvesting System Review. Biosystems Engineering, 2005, 90(2): 115125

Shiqchik Hayashi, Katsunobu Ganno, Yukitsugu Ishii, et al. Robotic Harvesting System for Eggplants. JARQ, 2000, 36(3): 163-168

Stephen Westland and Caterina Ripamont. Computational Colour Science using Matlab. John Wiley \& Sons. Ltd, 2003, 2

Tadhg Brosnan, Da-Wen Sun. Improving quality inspection of food products by computer vision-a review. Journal of Food Engineering, 61 (2004):3-16

W.S. Lee, D.C. Slaughter, and D.K. Giles. Robotic weed Control System for Tomatoes. Precision Agricultural, 1 (1999): 95-133

Yud-Ren Chen, Kuaiglin Chao, Moon S. Kim. Machine Vision technology for agricultural application. Computer and Electronics in Agriculture, 36 (2002):173-191 